\title{
Facilitated Loading of Horizontal Gels Using a Capillary Comb Loader
}

BioTechniques 34:814-818 (April 2003)

\author{
J. Thompson, T. Pugh, \\ K. Guggenheimer, A. Safar- \\ pour, A. Christie, J. Pel, \\ S. Chow, and A. Marziali \\ University of British Columbia, \\ Vancouver, BC, Canada
}

\begin{abstract}
Manual loading of samples into horizontal gels, such as the agarose gels commonly used for DNA fragment sizing and quantification, is laborious and prone to errors. Manual-loading times for highthroughput gels can reach $10 \mathrm{~min} / \mathrm{gel}$, and human error can result in incorrect identification of samples because of reverse loading or other errors in the loading process. To reduce gel-loading times and to improve reliability, a novel comb has been developed that uses glass capillaries and hydrostatic pressure to simplify sample loading from microplates. Accurate sample metering is ensured by the uniform length and volume of the capillaries. The loaded comb is placed in the gel boat over a pre-cast agarose gel, and buffer is added to a reservoir at the top of the comb. Once the buffer rises over the ends of the capillaries, the samples are pushed into the wells by hydrostatic pressure. This technique was successfully demonstrated for a 24-lane comb. This capillary comb loader reduces loading time, maintains well-to-well uniformity, and retains the same geometry and appearance of manually loaded bands, making this loading method compatible with existing downstream processes and software for subsequent analysis of the gel image.
\end{abstract}

\section{INTRODUCTION}

Agarose gels continue to be the dominant DNA fragment separation and analysis technique for many applications ranging from quantification of PCR products to high-throughput separations for bacterial artificial chromosome (BAC) fingerprinting (1) where several thousand samples are loaded per batch of gels. The latter is of particular interest because of the high-throughput BAC fingerprinting activities of the local genome center (BC Cancer Agency Genome Sciences Center). Currently, technicians load these high-capacity gels manually using 8- or 12-channel syringes or pipettors, targeting sample wells as small as $1 \times 1 \mathrm{~mm}$. Wells are typically loaded with a $1.5 \mu \mathrm{L}$ mixture of sample and loading dye. This is a technique that requires training and time (approximately $10 \mathrm{~min} / \mathrm{gel}$ box for an experienced technician). Furthermore, repeatability and data quality can vary among technicians. Automation of this loading process would lead to significant labor savings, reduced loading errors, and improved data quality.

Previous attempts have been made at automated gel loading. Pneumatic gel loaders have been useful for loading acrylamide sequencing gels (2) but are not appropriate for accurately metering loaded volume when the samples are drawn from a plate containing more sample than is to be loaded into the gel. Direct loading of agarose gels from capillaries containing sample has also been demonstrated (3). In the latter case, the capillaries are stabbed into the gel to form the loading well and to in- ject samples. Variability in loaded amounts has been identified as a drawback to this technique. Furthermore, the bands resulting from electrophoresis of samples injected in this fashion appear to retain the irregular geometry of the holes formed by the capillariesthis is a potential problem for some image analysis software such as that used for automated sizing of BAC fingerprint samples. Robotic loading of gels using a liquid-handling robot remains a possibility, though the poor positional repeatability of the wells formed in agarose gels may require the use of machine vision for accurate loading. In any case, because of the large format $(40 \times 23 \mathrm{~cm})$ of the high-throughput gels typically adopted for BAC fingerprinting, a robotic solution would be large, cumbersome, and expensive.

The sample loading approach described here is based on flexible glass capillaries with an internal diameter of $180 \mu \mathrm{m}$. The flexibility of the capillaries allows reformatting of the sample geometry from multiple rows of a microplate into a linear format compatible with high-density agarose gels. The samples are loaded directly from a microplate into one end of the capillary array by pneumatic pressure and are unloaded from the opposite end of the capillaries into the wells of a gel by the hydrostatic pressure of a column of buffer. This novel approach eliminates the tedious and repetitive pipetting between the microplate and the gel and provides consistent well-to-well sample loading uniformity. The use of the running buffer to unload the samples eliminates unwanted air bubbles often introduced into the 
wells during manual loading. Furthermore, a standard gel comb is used to cast the wells, and the loaded samples have a well geometry identical to that generated using manual methods.

The capillary gel comb is composed of two functional parts: the loading end and the unloading end. Glass capillaries extend from one end of the gel comb to the opposite end; their length determines the volume of the sample to be loaded. At the unloading end of the comb, the glass capillaries are positioned linearly at intervals corresponding to the distance between the teeth of the standard gel comb that is used to cast the wells. In this first prototype, 24 capillaries are positioned $4.5 \mathrm{~mm}$ apart in a straight line. The capillaries of the comb in this arrangement align with the teeth of the 26-lane comb. Each glass capillary is protected by a piece of stainless steel tubing at the unloading end and extends above an alignment plate to a loading region. At the loading end of the comb, the alignment plate re-arrays the glass capillaries into two rows with $9 \mathrm{~mm}$ spacing. This arrangement corresponds to two rows (24 wells) of a 96well microplate. The same approach can be extended to capillary combs capable of loading 96 samples at a time, from a single 96-well microplate.

The glass capillaries on the loading end of the comb protrude sufficiently below the alignment plate to allow them to be inserted directly into a microplate. To load the samples, the microplate or a portion of the plate (in the form of PCR strips) is placed in a loading chamber. The empty capillaries of the comb are then immersed into the wells of the microplate containing samples pre-mixed with loading dye. Upon contact, the samples are initially aspirated by capillary action; to completely fill the capillaries, however, a squeeze bulb is connected to the loading chamber to provide positive pressure.

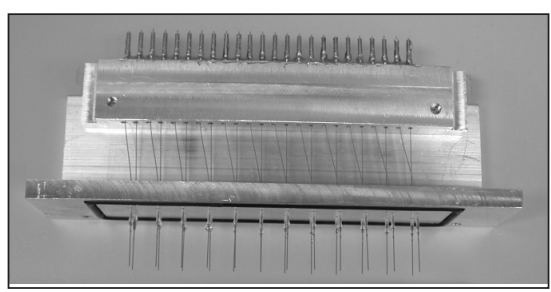

Figure 1. 24-channel comb loader.
Once the capillaries are filled, the comb is inverted and placed on the gel boat containing a previously cast gel. In this position, the loading ends of the capillaries are facing up, and the unloading ends of the capillaries are positioned within the wells of the gel. To unload the samples, a reservoir equipped with a column is attached to the loading end of the comb. Running buffer is poured into this column to provide the hydrostatic pressure required to unload the samples. When the level of the buffer in the column reservoir rises above the upper ends of the capillaries and into the column, hydrostatic pressure pushes the samples out of the capillaries and into the wells of the gel. Because the density of the sample is greater than that of the buffer, the samples settle into the bottom of the wells. The running buffer present in the comb reservoir will also enter the wells through the capillaries, ensuring that no air bubbles are formed. The low hydrostatic pressure created by the buffer column (less than $2 \mathrm{kPa}$ ) ensures that the running buffer flows at a low enough rate to leave the samples undisturbed.

Between uses, the capillary comb is washed with distilled water and dried under vacuum. A loading/washing chamber attached to a vacuum pump is used for this process. Because of the small volumes of the capillary lengths

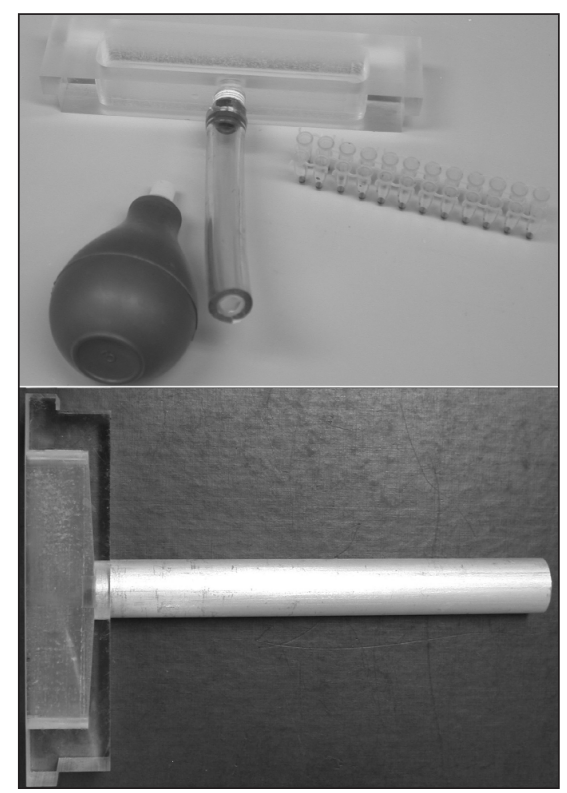

Figure 2. Loading reservoir (top) and unloading reservoir (bottom). being used, only a short cleanup time is necessary to cleanse the system thoroughly. The total time required to load a gel, including cleanup, is about $70 \mathrm{~s}$.

\section{MATERIALS AND METHODS}

\section{Capillary Comb Construction}

The comb loader prototype is machined out of three pieces of 6061 aluminium (Figure 1). The top piece, which constitutes the unloading end, has 24 holes approximately $1.8 \mathrm{~mm}$ in diameter spaced $4.5 \mathrm{~mm}$ apart. In each hole, 25-mm-long sections of stainless steel tubing with an internal diameter of $0.5 \mathrm{~mm}$ and an external diameter of $1.59 \mathrm{~mm}$ (Small Parts, Miami Lakes, FL, USA) were inserted and secured in place with epoxy. The bottom piece in Figure 1 is the alignment plate and constitutes the loading end of the comb. In this piece, 24 holes, approximately 1.8 $\mathrm{mm}$ in diameter, are drilled in two rows of 12 holes on a 9-mm square grid. In each of these holes, a plastic P-30 $\mu \mathrm{L}$ pipet tip, (Matrix Technologies, Hudson, NH, USA) cut at approximately two-fifths of its length, is inserted and secured with epoxy to act eventually as a sheath for the capillaries. An o-ring groove is machined into the bottom piece to allow the loader to create a seal with the reservoirs.

Once the loader is assembled, with steel tubing and pipet tips in place, glass capillaries with an internal diameter of $180 \mu \mathrm{m}$ and an external diameter of $337 \mu \mathrm{m}$ were cut to $91-\mathrm{mm}$ lengths (for a $2.3-\mu \mathrm{L}$ sample volume). These are inserted into the steel tubing and threaded through to the bottom of the pipet tips. Approximately $20 \mathrm{~mm}$ of capillary extends beyond the pipet tips, and between 0 and $1 \mathrm{~mm}$ extends beyond the steel tubing.

Two acrylic reservoirs are used in combination with the comb loader. One reservoir is used for loading the samples and cleaning the capillaries, and the other is used for unloading the samples. The loading reservoir (Figure 2, top) consists of a piece of acrylic with a $120 \times 22 \mathrm{~mm}$ cavity machined to a depth of approximately $23 \mathrm{~mm}$, designed to house two rows of a PCR plate (CyclePlate ${ }^{\circledR}$-96ET; Robbins Sci- 


\section{Research Report}

entific, Sunnyvale, CA, USA) containing the sample to be loaded. On one side of the reservoir, a hole is drilled and tapped to $1 / 4$ NPT pipe thread, and a fitting secured to a piece of tubing is attached. In the unloading reservoir (Figure 2 , bottom), a $120 \times 22 \mathrm{~mm}$ cavity is machined to a depth of approximately $30 \mathrm{~mm}$. The top of the cavity in the unloading reservoir is cut at an angle of approximately $3^{\circ}$, sloping towards the center of the reservoir to allow air bubbles to escape when filling the reservoir with buffer solution. At the center of the cavity, a $25-\mathrm{mm}$ diameter hole is drilled and a piece of aluminum tubing approximately $20 \mathrm{~cm}$ long is inserted flush with the bottom of the hole and fixed in place with epoxy.

\section{Loading and Unloading Protocol}

To load the samples, a strip of 24 wells (two rows) from the 96-well plate is placed into the loading chamber. The comb is then inverted, and the capillaries protruding from the inverted comb are inserted into the two rows of wells. Two 1-in fold-back paper clips are used to secure the comb tightly in place and to form a seal with the loading chamber. A squeeze bulb is connected to the loading chamber through a piece of tubing. The assembled loading apparatus is shown to the left in Figure 3. To load the samples up to the tips of the capillaries, the bulb is squeezed until the operator sees that the capillaries are filled and then disconnected from the tubing. The clips are then removed, and the loading chamber is replaced with the buffer chamber, equipped with a 20 -cm-long open column, having an internal diameter of $20 \mathrm{~mm}$. A $1 \%$ agarose solution in
1× TAE (40 mM Tris-acetate, $\mathrm{pH} 8.0,1$ $\mathrm{mM}$ EDTA), containing $0.5 \mu \mathrm{g} / \mathrm{mL}$ ethidium bromide, is previously poured into an electrophoresis boat, while a 26lane comb (WVR international, Mississauga, ON, Canada) is used to cast the wells. Once the buffer chamber is mounted to the capillary comb loader and secured tightly in place with the clips, the resulting assembly is mounted in the electrophoresis tray and aligned with the gel wells. The assembled unloading apparatus mounted in the gel boat is shown to the right in Figure 3 . $1 \times$ TAE buffer, having already been poured over the gel in the electrophoresis apparatus, is subsequently poured into the buffer reservoir sitting on top of the comb. As the level of the buffer in the loader reservoir rises above the tips of the capillaries, the samples start to unload because of hydrostatic pressure created by the column of buffer as seen in Figure 4. Once all of the capillaries have unloaded, the capillary gel comb is removed from the tray and electrophoresis can begin.

\section{Loading Sample Control- 1-kb Ladder}

To verify the performance of the loader with DNA, a 1-kb ladder was loaded and run on a gel. One hundred microliters of $1-\mathrm{kb}$ ladder at $500 \mu \mathrm{g} / \mathrm{mL}$ (New England Biolabs, Pickering, ON, Canada) is mixed with $100 \mu \mathrm{L} 6 \times$ DNA loading buffer $(0.25 \%$ bromophenol blue, $0.25 \%$ xylene cyanol FF, $30 \%$ glycerol, $6 \times \mathrm{TAE}$ ) and $400 \mu \mathrm{L}$ water. Six or seven microliters of the resulting sample are transferred into 24 wells of a 96-well microplate (CyclePlate-96ET) and are kept at $4^{\circ} \mathrm{C}$ until ready to load.

\section{Cleaning the Comb}

To wash the comb, the capillaries at the loading end of the comb are placed into the washing chamber, an attachment similar to the loading chamber. The chamber is connected to a vacuum pump through a section of tubing and a pipefitting. Once the pump is running and a vacuum is created in the chamber, the tips of the capillaries at the unloading end are dipped for $5 \mathrm{~s}$ into a tray containing distilled water. Water drawn through the capillaries accumulates in the washing chamber. After a further 10 $\mathrm{s}$ of aspirating drying air, the vacuum pump is stopped and the accumulated water is discarded. To load samples effectively, the capillaries must be clean and free of water droplets.

\section{RESULTS AND DISCUSSION}

A 24-lane prototype capillary comb has been manufactured as described in the Materials and Methods section. This comb loads samples directly from two rows of a 96-well format microplate and unloads the samples into 24 wells in a 14-cm-wide agarose gel. To accommodate the different arrangements of wells in a microplate and those in a 24-lane gel, the loading ends of the capillaries are arrayed into two rows of 12 capillaries at $9 \mathrm{~mm}$ spacing, appropriate for loading from a 96-well plate. These capillaries are then reorganized in a straight line with $4.5-\mathrm{mm}$ intervals at the unloading end, corresponding to 24 wells of a 26-lane comb.

Using the protocol described in the Material and Methods section, we have utilized the prototype comb to load

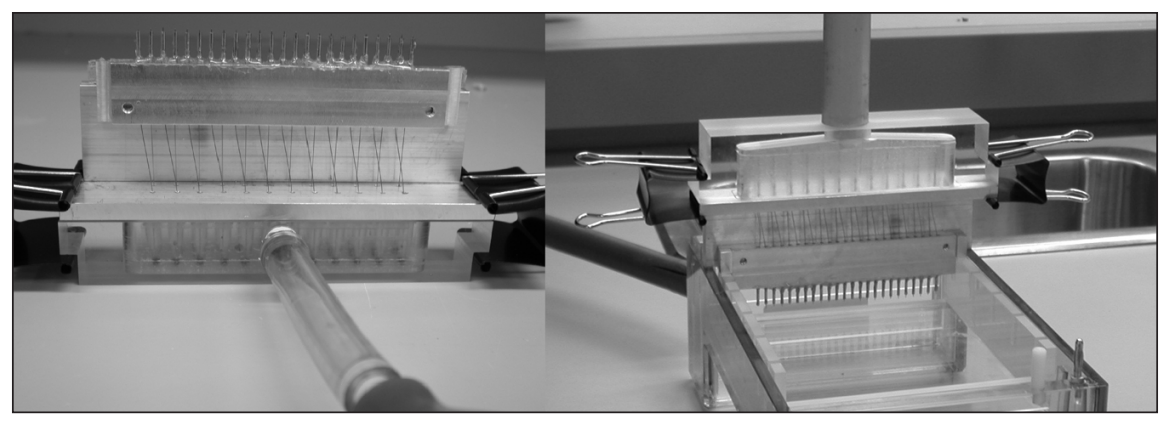

Figure 3. Comb loader in loading (left) and unloading (right) positions. Left: capillary comb inverted for loading from two rows of microplate in the loading chamber. Right: capillary comb with unloading reservoir attached.

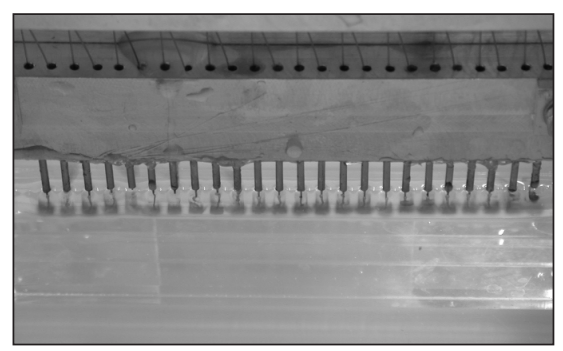

Figure 4. Unloading of the sample into $1 \%$ agarose gel. As the level of buffer in the unloading reservoir increases, the samples start to unload from the capillaries of the gel comb into the corresponding wells in the agarose gel. 


\section{Research Report}

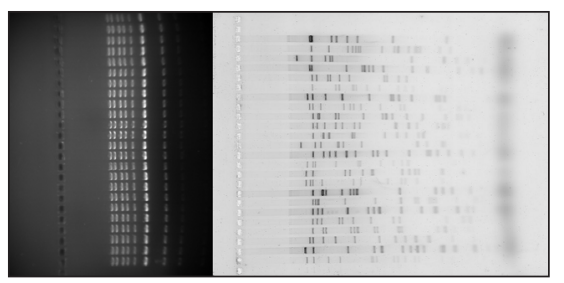

Figure 5. Gels loaded with the capillary comb. Left: identical 1-kb ladder samples loaded with the capillary comb and the resulting gel image after electrophoresis. Note the uniform sample intensity and band geometry obtained in the gel image. Right: image of a BAC fingerprinting gel, after electrophoresis, that was loaded with the capillary comb and run at the BC Cancer Agency Genome Sciences Center. (Image courtesy of BC Cancer Agency Genome Sciences Center)

more than 60 test gels using standard loading dye. Direct loading of the comb from a microplate and subsequent transfer of sample to the gel were successful for all of the samples, with the exception of one gel in which a single capillary failed to dispense sample. The source of the failure was found to be an agarose plug formed in the capillary when it was accidentally stuck directly into the gel rather than into the target well. Similar tests were run with comparable success using the $1-\mathrm{kb}$ ladder sample control, and little difference in fluidic behavior from the pure dye trials was observed. Gel images obtained after electrophoresis of samples loaded with 1-kb DNA ladder and BAC fingerprint samples indicate that there is no noticeable difference between a gel loaded with the prototype capillary comb and a gel loaded manually by an experienced technician.

To identify qualitatively any lane-tolane variation in amount of sample loaded with the capillary comb, 1-kb ladder samples from a single run (24 samples) on a gel were compared. The left image of Figure 5 shows the uniformity of the resulting image after electrophoresis. There is no significant variability in intensity between lanes. The low flow rate at which samples, and subsequently buffer, unload from the capillaries of the comb ensures that sample present in the well is not disturbed by the buffer flow. Independently prepared DNA samples loaded with the capillary comb showed good signal strength and resolution (Figure 5, right). Any sample lost during loading was minimal. The signal strength of DNA ladder loaded with the comb loader was within $10 \%$ of the signal strength of $2 \mu \mathrm{L}$ manually loaded ladder (data not shown).

The time required to load a gel using the capillary comb is approximately 70 $\mathrm{s}$ including $30 \mathrm{~s}$ of washing and drying the comb. Though this is comparable to manual loading times for a 24-lane gel, the time required for loading larger gels increases linearly with the number of wells for manual methods, while the time required to use the capillary comb loader is expected to be independent of the number of wells in the comb. Furthermore, the capillary comb loads all samples in a single operation, decreasing the risk of reverse loading and similar manual errors that occasionally result in corrupted sample data.

In summary, a capillary gel comb that allows direct loading of samples from a microplate into a horizontal agarose gel has been constructed and tested with excellent results. The comb currently takes the form of a reusable 24-channel device intended to load two rows of a 96-well plate into 24 gel lanes with $4.5 \mathrm{~mm}$ spacing. Different comb formats based on the design presented are possible by changing the comb tooth spacing and the number of rows of microplate to be loaded with each comb. A 96-channel device that loads directly from a 96-well microplate is currently in development.

\section{ACKNOWLEDGMENTS}

Special thanks goes out to the staff of the BC Cancer Agency Genome Sciences Centre for their support in fieldtesting of our device. This work is supported in part by GenomeBC and by NHGRI (HG02072-03).

\section{REFERENCES}

1.Marra, M.A., T.A. Kucaba, N.L. Dietrich, E.D. Green, B. Brownstein, R.K. Wilson, K.M. McDonald, L.W. Hillier, J.D. McPherson, and R.H. Waterston. 1997. High throughput fingerprint analysis of largeinsert clones. Genome Res. 7:1072-1084.

2.Panussis, D.A., M.W. Cook, L.L. Rifkin, J.E. Snider, J.T. Strong, R.M. McGrane, R.K. Wilson, and E.R. Mardis. 1998. A pneumatic device for rapid loading of DNA sequencing gels. Genome Res. 8:543-548.

3.Evensen, H.T., D.R. Meldrum, C. Saenphimmachak, and E.E. Dixon. 1999. Highdensity small-volume gel loading directly from capillary tubes. BioTechniques 27:974978.

Received 5 December 2002; accepted 23 January 2003.

Address correspondence to:

Dr. Andre Marziali

Department of Physics and Astronomy

6224 Agricultural Road

University of British Columbia

Vancouver, BC, Canada, V6T 1Z1

e-mail: andre@physics.ubc.ca 